\title{
Pobreza y marginalidad como criterio de priorización para las enfermedades catastróficas en México
}

\author{
Poverty and marginality as prioritization criteria for \\ catastrophic diseases in Mexico
}

\author{
Hilda Santos Padrón \\ Dra.C. de la Salud. Instituo de Seguridad Social de Tabasco (ISSET), México.
}

\section{RESUMEN}

Introducción En una de las etapas de la investigación titulada "Modelo para evaluar la sustentabilidad del Fondo de Protección contra gastos catastróficos en México. 2005-2010", se desarrolló un proceso de priorización de 30 enfermedades catastróficas seleccionadas de la Lista Oficial elaborada por el Consejo General de Salud en México. El proceso de priorización se basó en cuatro criterios: de trascendencia, pérdida social, marginalidad y capacidad de respuesta institucional. En este trabajo sólo se describe lo relacionado con la marginalidad.

Objetivos Describir el cálculo y aplicación del criterio de marginalidad dentro del proceso de priorización y mostrar los resultados de su aplicación.

Métodos La metodología incluyó la utilización del Índice de Desarrollo Humano, obtenido para cada una de las 32 entidades federativas mexicanas y el cálculo del índice de correlación entre el de Desarrollo Humano y las tasas de mortalidad, para las 30 enfermedades catastróficas, en esos territorios en el año 2004.

Resultados Los resultados mostraron moderada correlación entre las entidades con menor desarrollo socio-económico y tasas elevadas de enfermedades catastróficas, especialmente para el cáncer cérvico-uterino, enfermedad no incluida para su financiamiento por el Fondo. Se consideró novedoso el proceso de priorización en su conjunto, como contribución al Sistema de Protección Social en Salud en México y el cálculo del criterio de marginalidad, para la detección de las desigualdades territoriales.

Conclusiones Se reconoce la importancia de reorientar los modelos de reforma de salud hacia estrategias socialmente enfocadas, que beneficien a poblaciones marginadas o empobrecidas y que dediquen recursos a esas entidades menos favorecidas. 
Palabras clave: Criterio de priorización, pobreza, marginalidad, reforma de servicios de salud, protección social en salud.

\begin{abstract}
Introduction In one of the stages of the research entitled Model for evaluation of sustainability of the Protection Fund against Catastrophic Expenditures in Mexico, 2005-2010, a process of prioritization of 30 catastrophic diseases taken from the Mexican General Health Council Official List was developed. This process was based on four criteria: significance, social loss, marginality and institutional response capacities. This paper described those aspects related to marginality.

Objectives To describe the calculation and application of the marginality criterion within the prioritization process and to show the final results.

Methods The methodology involved the use of the Human Development Index for each of the 32 Mexican federative entities and the estimation of the correlation index between the Human Development Index and the mortality rates of the 30 catastrophic diseases in the year 2004.

Results The results showed a moderate correlation between the federative entities with lower socioeconomic development and higher rates of catastrophic diseases, particularly for cervix and uterine cancer, a disease that is not eligible for financing by the Protection Fund. The process of prioritization as a contribution to the System of Social Protection in Health in Mexico and also calculation of the marginality criterion for the detection of territorial inequalities were both considered novelties. Conclusions The importance of re-directing the health reform models to sociallyfocused strategies that benefit marginalized or impoverished populations and of devoting some resources to those less favoured entities was recognized.
\end{abstract}

Key words: Prioritization criterion, poverty, marginality, health service reform, social protection in health.

\title{
I NTRODUCCI ÓN
}

Las relaciones de la pobreza y la salud -en el campo estrictamente sanitario- se remonta a los inicios de la medicina moderna y al nacimiento mismo de la salud pública como ciencia, cuando reconocidos precursores como Virchow, Villermé, Snow y Farr popularizaron la idea de que la pobreza y las malas condiciones de vida eran causas de las enfermedades, de la mayor mortalidad y de la menor esperanza de vida de su época. Uno de los trabajos que recoge esta idea básica de la medicina social, fue el informe de Chadwick (1842) relativo a las condiciones de salud de la clase trabajadora en la Inglaterra de la revolución industrial. ${ }^{1}$ Asombra que, traspasado el umbral del siglo xx en la Cumbre del Milenio de las Naciones Unidas, los líderes mundiales de 189 estados recrean esta relación salud-pobreza, al adoptar un conjunto de objetivos y metas cuantificables y delimitadas en tiempo para combatir la pobreza, el hambre, las enfermedades, el analfabetismo, la degradación del medio ambiente, la discriminación contra la mujer, y comprometiéndose con los principios de los derechos humanos, la gobernanza responsable y la democracia. Estos objetivos y metas, denominados Objetivos de 
Desarrollo del Milenio (ODM) a alcanzarse para el año 2015, se resumieron en un paquete de ocho objetivos generales, 18 metas y 48 indicadores y, para las Américas, el énfasis en la reducción de las desigualdades, en particular las de salud, es de especial importancia para lograr los ODM. ${ }^{2}$

Recientemente, la máxima autoridad sanitaria mundial, ha enfatizado la interacción entre el nivel de salud de una población y reconoce que "la pobreza es el principal obstáculo al desarrollo y al cambio, y la responsable de la mayor parte de las muertes por causas evitables" (Declaración con motivo del Día de la Mujer, 2007); también sostiene que "las poblaciones subatendidas siguen sin poder contar con una atención sostenible, equitativa e integral que se pueda dispensar a escala suficiente" y concluye señalando que "si queremos que la mejora de la salud funcione como estrategia de reducción de la pobreza, hemos de llegar a los pobres. Es ahí donde fallamos" (Discurso de Apertura ante la Conferencia Internacional de Salud para el Desarrollo, 2007).

Con anterioridad a estos planteamientos, ya en la década de los años 90 del pasado siglo, las reformas más recientes de los sistemas de salud en los países latinoamericanos, incluido México, pretendieron universalizar el acceso al conjunto mínimo de servicios de salud, que garantizara a todos los ciudadanos la preservación de su estado de salud o su restitución cuando los afecte alguna enfermedad. Las reformas establecen mecanismos de acceso a los servicios de más alto costo, en magnitud no lograda en países más desarrollados y, para garantizar la sustentabilidad y, consecuentemente, la permanencia de estos mecanismos públicos, países como Chile y México han estudiado y desarrollado diversas estrategias que prioricen la acción pública, para que los recursos alcancen a los grupos de población más vulnerables.

Según Wyszewianski, "las enfermedades de alto costo o catastróficas, pueden definirse de diversas maneras y desde el punto de vista financiero, representa una enfermedad cuyo tratamiento implica un desembolso monetario importante y que excede algún umbral considerado normal. ${ }^{13}$ Estos padecimientos, generalmente, impactan con fuerza en el presupuesto familiar y, eventualmente, dejan a las familias en un estado de insolvencia financiera temporal o definitiva. Existe la convención de que una enfermedad, tiene un impacto catastrófico, cuando para su atención se destina más del $30 \%$ del presupuesto familiar. ${ }^{4}$ En ese sentido, resultan trascendentes esas estrategias sanitarias que "priorizan la acción pública" pues, existe la equivocada creencia de que estas enfermedades, generalmente, se considera como de alta incidencia en las sociedades más desarrolladas y, por contraste, de menor prioridad en el interés público, lo que disminuye la necesidad de implementar acciones urgentes para detener su crecimiento entre la población, sobre todo, para contener el rápido incremento de su prevalencia entre los menos favorecidos.

\section{Antecedentes}

Es innegable que las ideas de la medicina social, tuvieron un impacto significativo en las políticas sanitarias de la segunda mitad del siglo xix y originaron algunas medidas de gran trascendencia, como la imposición del seguro social obligatorio en Alemania, sin embargo, otros avances científicos en ese propio siglo xix e inicios del $x x$ contribuyeron -entre otros elementos- a que el enfoque social se soslayara, sustituido por las teorías contagionistas acerca de la enfermedad. No obstante, las contribuciones de Chadwick a la economía de la salud, resultaron muy avanzadas para su época y adelantaron el enfoque de las políticas sanitarias del siglo xx, así como los instrumentos para su diseño, financiamiento y ejecución. Cierta tendencia al reduccionismo al interpretar la propuesta avanzada de Chadwick, provocó que las 
políticas públicas de su época se centrara en eliminar la inmundicia, construir redes de alcantarillado para las aguas residuales e instalar fuentes de agua potable y relegara a la pobreza como tal, la desnutrición y las malas condiciones de vida como objetivos sobre los cuales orientar la acción pública.

En la tercera y cuarta década del siglo xx, se recuperó y amplió el planteamiento original de la medicina social, con los renovados fundamentos de la higiene social, aunque también casi restringidos al continente europeo. Es en la década del 70 del propio siglo xx que en la Declaración de Alma Atá, se recrea universalmente el tema de la equidad en salud, al proponerse una estrategia de atención primaria a la salud, de fuertes implicaciones sociopolíticas. Se reclamaba una distribución más justa de los recursos, tanto al interior de los países, como entre las naciones con diferentes grados de desarrollo, mediante el compromiso político de aumentar los presupuestos nacionales destinados a la salud, hasta posibilitar que toda la población tuviera acceso a los servicios. En esa estrategia de atención primaria a la salud, también se reconocía la estrecha asociación entre salud y desarrollo en los sectores más empobrecidos de las sociedades, y se establecía que no es defendible ninguna distinción entre desarrollo social y económico, lo que se reforzaba al determinar que los factores sociales son, precisamente, la fuerza impulsora del desarrollo. ${ }^{5}$

Por otra parte, la generación de reformas aplicadas a los sistemas de salud en las últimas tres décadas, de manera particular en los países latinoamericanos, ha perseguido tanto la mejora del desempeño de dichos sistemas, como el fortalecimiento de los principios que guían a la salud pública moderna. Lo que cambia, según Bengoa, son "las formas de organizar y gestionar el sector para alcanzar esos principios y fines sociales". Para este autor cualquier reforma debe preservar, sobre todo, el objetivo estratégico de la equidad, o sea, el derecho a acceder a servicios básicos de salud, que tiene todo ciudadano cuando afronta una condición sanitaria específica. ${ }^{6}$

Con estos antecedentes, la reforma al sistema de salud mexicano, aplicada a partir de 2004, se propuso la introducción de cambios significativos en materia de cobertura, protección financiera y organización de los servicios, para el acceso universal de los mexicanos a un conjunto de intervenciones médico-quirúrgicas, farmacéuticas y hospitalarias que satisfagan de manera integral sus necesidades de salud. Este rediseño, sustentado en las acciones del nuevo Sistema de Protección Social en Salud (SPSS), comprende tanto intervenciones de carácter preventivo y de promoción de la salud, como acciones dirigidas a la atención de las personas, mediante dos modalidades de acceso: atención de las necesidades esenciales en salud y atención a las enfermedades que ocasionan gastos catastróficos.

Con respecto a las enfermedades catastróficas (EC), el SPSS tiene como meta subyacente evitar el empobrecimiento -en términos absolutos- debido a los desembolsos que las generan; resolver no solamente los retos que plantean, la complejidad y el riesgo biológico que afecta a la persona enferma, sino también el riesgo financiero que le acompaña y causa estragos a la estabilidad de su familia. ${ }^{7}$ Los resultados de una encuesta realizada en un estado de los Estados Unidos de América, muestran que el $19 \%$ de la población afectada por una EC tuvo necesidad de vender artículos personales para hacer frente a los gastos de atención y que el $16 \%$ de la población fue incapaz de afrontar dichos gastos. ${ }^{8}$

En ese sentido, el modelo de atención para las EC -título tercero bis de la Ley General de Salud de México-, supone el financiamiento solidario, mediante recursos provenientes del estado nacional y las entidades federativas, mediante una aportación equivalente al $8 \%$ de las cuotas y aportaciones que corresponden a 
estos órdenes de gobierno dentro del SPSS y que se destinan a la integración de un fondo común: el Fondo de Protección contra Gastos Catastróficos (FPGC). ${ }^{9}$

Asimismo, la mencionada Ley confiere al Consejo de Salubridad General, órgano superior del sistema de salud mexicano, la facultad para establecer la Lista Oficial de enfermedades que deben considerarse como causantes de gastos catastróficos y cuyo tratamiento es, por tanto, susceptible de financiarse con los recursos del citado Fondo. Además, este ordenamiento y su reglamentación secundaria disponen el establecimiento de un orden de prioridades que contribuya a la eficiencia y racionalidad en el gasto sanitario.

No obstante estas disposiciones, el SPSS inició sus operaciones sin un estudio riguroso para demostrar la sustentabilidad financiera, social, técnica e institucional del Fondo, con la relevante carencia de instrumentos tecnológicos como, un modelo de priorización para seleccionar las EC que debían financiarse. Esta falencia del SPSS, estimuló el diseño y ejecución de una investigación de desarrollo tecnológico realizada por la autora de este artículo, acerca de la sustentabilidad del Fondo, basada en las técnicas de la prospectiva. En una de sus etapas, se desarrolló un modelo de priorización para las EC que, a juicio de sus autores, es susceptible de adaptarse y utilizarse por las autoridades sanitarias mexicanas. Para construir el modelo, se priorizaron 30 de esas enfermedades según criterios de trascendencia, pérdida social, marginalidad y capacidad de respuesta institucional. En este trabajo se describe el cálculo y aplicación del criterio de marginalidad, dentro de ese proceso de priorización, así como se muestra los resultados de su aplicación.

\section{MÉTODOS}

A los fines de la investigación en que se sustenta el trabajo, se redujo la Lista Oficial de $50 \mathrm{EC}$ a 30 , se incluyeron procedimientos terapéuticos como los trasplantes, no registrados en el Sistema Estadístico Epidemiológico de Defunciones (SEED) y también los politraumatismos, contenidos en conglomerados de enfermedades que, además de generar un sub-registro en las estadísticas oficiales, limita la realización de análisis epidemiológicos más certeros.

Se definió la marginalidad, como la tendencia de las distintas enfermedades a acumularse en los estratos poblacionales menos favorecidos. El índice se obtuvo al cotejar la mortalidad, registrada por cada una de las 30 EC, con las disparidades de desarrollo regional, existentes en las 32 entidades federales que conforman la República Mexicana. Se añadieron, además, dos condiciones esenciales relacionadas con los datos utilizados:

\section{Que correspondieran al año 2004, por ser el año de inicio del SPSS, y}

2. que estuvieran referidos a la población abierta, o sea, a la población carente de seguridad social, susceptible de ser cubierta por el Fondo de Protección contra Gastos Catastróficos.

Para calcular las tasas crudas de mortalidad, se revisó la base de datos del SEED de la Secretaría de Salud. Se obtuvo así el número de defunciones registradas en el año 2004 por cada una de las 30 EC, correspondientes a la población no asegurada y el lugar de residencia habitual de esos fallecidos. También se recopilaron los datos sobre el Índice de Desarrollo Humano (IDH), indicador establecido por el Programa de las Naciones Unidas para el Desarrollo (PNUD) para comparar el nivel de bienestar entre países, ${ }^{10}$ en las 32 entidades federativas en el mismo año 2004. 
De los datos sobre la mortalidad y el IDH estatal, se elaboraron los gráficos de dispersión y finalmente, se calculó el coeficiente de correlación de Pearson para cada una de las enfermedades. El coeficiente de correlación $(r)^{11}$ es un índice estadístico que mide la relación lineal entre dos variables cuantitativas, en este caso, IDH y tasa cruda de mortalidad; su valor mide la fuerza de esta relación lineal y varía entre -1 y +1 . Los resultados, se explicaron de la forma siguiente:

Si $r=0$, no existe ninguna correlación.

Si $r=1$, existe una correlación positiva perfecta.

Si $0<r<1$, existe una correlación positiva

-Si - $1<r<0$, existe una correlación negativa.

\section{RESULTADOS}

Al describir las tasas de mortalidad por EC como uno de los indicadores para el cálculo de correlación (tabla 1 ) se advierte que, al infarto agudo del miocardio $(16,4)$, el cáncer cérvico-uterino $(5,9)$ y la cirrosis hepática $(4,8)$, les correspondieron las más elevadas, sin embargo, cuando se aplicó el criterio de marginalidad en las 30 EC (tabla 2), esta situación se modificó y alcanzaron los más elevados índices de correlación la insuficiencia renal crónica $(-0,476)$, la artritis reumatoide $(-0,456)$ y el cáncer cérvico-uterino $(-0,414)$. Para las entidades de mayor desarrollo -IDH elevado- predominaron el cáncer de mama $(0,30)$, el $\mathrm{VIH} / \mathrm{SIDA}(0,31)$ y los defectos diafragmáticos en recién nacidos $(0,24)$. Resultó llamativo, que ninguna de estas EC, se incluyeron entre las que reciben financiamiento del Fondo.

Tabla 1. Mortalidad por enfermedades catastróficas. México, 2004

\begin{tabular}{|l|l|l||c|c|}
\hline \multicolumn{2}{|c|}{ Clave(S) CIE10 } & Enfermedad catastrófica & Casos & $\begin{array}{c}\text { Tasa de } \\
\text { mortalidad }\end{array}$ \\
\hline \hline 1 & 121 & $\begin{array}{l}\text { Infarto agudo del } \\
\text { miocardio }\end{array}$ & $\begin{array}{c}17 \\
372\end{array}$ & 16,49 \\
\hline 2 & C53 & Cáncer cérvico uterino (2) & 2261 & 5,92 \\
\hline 3 & K746 & Cirrosis hepática & 5060 & 4,80 \\
\hline 4 & C61X & Cáncer de próstata (3) & 741 & 2,92 \\
\hline 5 & C50 & Cáncer de mama (2) & 1073 & 2,81 \\
\hline 6 & M06 & Artritis reumatoide & 2281 & 2,17 \\
\hline 7 & C81 - C96 & Leucemia y linfomas & 2195 & 2,08 \\
\hline 8 & C34 & $\begin{array}{l}\text { Cáncer broncogénico y } \\
\text { pulmonar }\end{array}$ & 2146 & 2,04 \\
\hline 9 & B20-B21 & VIH/SIDA & 1992 & 1,89 \\
\hline 10 & T07X & Politraumatizado & 1636 & 1,55 \\
\hline 11 & E100-E110- & Coma diabético & 1269 & 1,20 \\
\hline
\end{tabular}




\begin{tabular}{|c|c|c|c|c|}
\hline & $\begin{array}{l}\text { E120-E130- } \\
\text { E140 }\end{array}$ & & & \\
\hline 12 & Q24 & $\begin{array}{l}\text { Cardiopatías congénitas } \\
\text { (1) }\end{array}$ & 2018 & 1,03 \\
\hline 13 & $\mathrm{C} 16$ & Cáncer de estómago & 1055 & 1,00 \\
\hline 14 & P36 & Sepsis neonatal ( 1 ) & 1594 & 0,81 \\
\hline 15 & C19X & Cáncer del colon y recto & 790 & 0,75 \\
\hline 16 & C56 & Cáncer de ovario (2) & 273 & 0,71 \\
\hline 17 & 161 & Hemorragia intraencefálica & 708 & 0,67 \\
\hline 18 & 163 & Infarto cerebral & 702 & 0,67 \\
\hline 19 & M32 & $\begin{array}{l}\text { Lupus eritematoso } \\
\text { sistémico }\end{array}$ & 646 & 0,61 \\
\hline 20 & P05-P07 & Prematurez (1) & 881 & 0,45 \\
\hline 21 & $\mathrm{P} 21$ & Asfixia neonatal (1) & 767 & 0,39 \\
\hline 22 & N188-N189 & Insuficiencia renal crónica & 277 & 0,26 \\
\hline 23 & P28 & $\begin{array}{l}\text { Otros problemas } \\
\text { respiratorios del recién } \\
\text { nacido (1) }\end{array}$ & 513 & 0,26 \\
\hline 24 & Q40 & $\begin{array}{l}\text { Malformaciones congénitas } \\
\text { del tubo digestivo (1) }\end{array}$ & 346 & 0,18 \\
\hline 25 & N180 & $\begin{array}{l}\text { Insuficiencia renal } \\
\text { terminal }\end{array}$ & 185 & 0,18 \\
\hline 26 & P285 & $\begin{array}{l}\text { Insuficiencia respiratoria } \\
\text { del recién nacido (1) }\end{array}$ & 199 & 0,10 \\
\hline 27 & Q79 & $\begin{array}{l}\text { Defectos diafragmáticos } \\
\text { (1) }\end{array}$ & 148 & 0,08 \\
\hline 28 & 169 & $\begin{array}{l}\text { Secuelas de accidente } \\
\text { vascular }\end{array}$ & 71 & 0,07 \\
\hline 29 & 120 & Angina de pecho inestable & 31 & 0,03 \\
\hline 30 & 160 & Hemorragia subaracnoidea & 29 & 0,03 \\
\hline
\end{tabular}

(1): tasa $\times$ menores de 1 año, (2): tasa $\times 100000$ mujeres mayores de 14 años, (3): tasa x 100000 hombres mayores de 14 años.

Fuentes: Secretaría de Salud, Instituto Mexicano del Seguro Social, International Agency for Research on Cancer, UNAM, Instituto Nacional de Salud Pública.

Para esclarecer el procedimiento de cálculo, se escogió el cáncer cérvico-uterino. En la tabla 3 se aprecia las altas tasas que correspondieron a los estados de Oaxaca, Puebla, Veracruz y Chiapas, con 3,66; 2,86; 2,78 y 1,90 defunciones por 10000 habitantes, respectivamente y, estos cuatro estados -por su IDH- se ubicaron en los lugares 31, 24, 27 y 32. Las menores tasas se localizaron en los estados de Nuevo León, Aguascalientes, Chihuahua y Jalisco, con 1,2; 1,9; 2,1 y 3,6 defunciones por 10000 habitantes respectivamente, entidades federativas que ocuparon los lugares 2, 5, 8 y 13, respectivamente.

Tabla 3. Calculo de correlación para cáncer cérvico-uterino 


\begin{tabular}{|c|c|c|c|c|c|}
\hline No. & Estado & $\begin{array}{c}\text { Indice } \\
(x)\end{array}$ & Defunciones & $\begin{array}{l}\text { Población } \\
\text { total }\end{array}$ & $\begin{array}{l}\text { Mortalidad } \\
\text { x } 10000 \\
\text { hab. (y) }\end{array}$ \\
\hline 1 & Aguascalientes & 0,827 & 2 & 1028279 & 0,19 \\
\hline 2 & Baja California & 0,827 & 12 & 2867630 & 0,42 \\
\hline 3 & $\begin{array}{l}\text { Baja California } \\
\text { Sur }\end{array}$ & 0,832 & 4 & 489669 & 0,82 \\
\hline 4 & Campeche & 0,824 & 4 & 763037 & 0,52 \\
\hline 5 & Coahuila & 0,711 & 15 & 2511114 & 0,60 \\
\hline 6 & Colima & 0,826 & 6 & 584068 & 1,03 \\
\hline 7 & Chiapas & 0,832 & 83 & 4357301 & 1,90 \\
\hline 8 & Chihuahua & 0,801 & 7 & 3373391 & 0,21 \\
\hline 9 & $\begin{array}{l}\text { Distrito } \\
\text { Federal }\end{array}$ & 0,884 & 41 & 8814123 & 0,47 \\
\hline 10 & Durango & 0,797 & 15 & 1549309 & 0,97 \\
\hline 11 & Guanajuato & 0,780 & 36 & 5027179 & 0,72 \\
\hline 12 & Guerrero & 0,770 & 48 & 3249559 & 1,48 \\
\hline 13 & Hidalgo & 0,733 & 11 & 2370735 & 0,46 \\
\hline 14 & Jalisco & 0,755 & 25 & 6758852 & 0,37 \\
\hline 15 & México & 0,802 & 54 & $\begin{array}{c}14447 \\
120\end{array}$ & 0,37 \\
\hline 16 & Michoacán & 0,746 & 55 & 4213737 & 1,31 \\
\hline 17 & Morelos & 0,789 & 22 & 1698232 & 1,30 \\
\hline 18 & Nayarit & 0,764 & 14 & 991142 & 1,41 \\
\hline 19 & Nuevo León & 0,848 & 5 & 4178145 & 0,12 \\
\hline 20 & Oaxaca & 0,720 & 135 & 3693497 & 3,66 \\
\hline 21 & Puebla & 0,763 & 157 & 5480844 & 2,86 \\
\hline 22 & Queretaro & 0,804 & 12 & 1572772 & 0,76 \\
\hline 23 & Quintana Roo & 0,829 & 5 & 1053084 & 0,47 \\
\hline 24 & $\begin{array}{l}\text { San Luis } \\
\text { Potosí }\end{array}$ & 0,775 & 16 & 2398690 & 0,67 \\
\hline 25 & Sinaloa & 0,782 & 10 & 2747467 & 0,36 \\
\hline 26 & Sonora & 0,819 & 42 & 2448839 & 1,72 \\
\hline 27 & Tabasco & 0,771 & 24 & 2045537 & 1,17 \\
\hline 28 & Tamaulipas & 0,815 & 17 & 3106529 & 0,55 \\
\hline 29 & Tlaxcala & 0,768 & 14 & 1055648 & 1,33 \\
\hline 30 & Veracruz & 0,750 & 202 & 7274772 & 2,78 \\
\hline 31 & Yucatán & 0,780 & 20 & 1784267 & 1,12 \\
\hline 32 & Zacatecas & 0,761 & 9 & 1415269 & 0,64 \\
\hline Total & & & 1,122 & 105349837 & 1,07 \\
\hline
\end{tabular}

Al estudiar el gráfico de dispersión entre mortalidad por esta causa y nivel de desarrollo (figura), se observa la clara tendencia hacia la disminución de la 
mortalidad, en la medida en que se incrementa el IDH, o por contraste, una tendencia de la mortalidad a acumularse en las zonas de mayor rezago económico.

A semejanza del estudio referido, el coeficiente obtenido para aplicar el criterio de marginalidad para el cáncer cérvico-uterino, fue de -0,414, es decir, una correlación inversa moderada, que mostró la tendencia de esta enfermedad para producir mayores efectos catastróficos, donde existe mayor población en condiciones de pobreza, lo cual también confirma los hallazgos de otros estudios.

\section{DISCUSION}

Los modelos de priorización, para su correcta aplicación del enfoque social y económico de los problemas de salud, deben reflejar apropiadamente el impacto de las enfermedades en los grupos económicamente menos favorecidos, así como los gastos de diagnóstico y tratamiento que ocasionan. Así se garantiza la eficiencia y la efectividad del gasto sanitario, válido tanto para sociedades en desarrollo como para las más desarrolladas

En diversas investigaciones, se relacionan las enfermedades con algunos factores derivados de la pobreza, como el analfabetismo, la residencia en áreas rurales, la inaccesibilidad a los servicios de salud o la carencia o deficiencia de los servicios públicos básicos. En otros, se utiliza el modelo de regresión de Poisson para estudiar este tipo de vinculación y así hallaron que el riesgo de enfermar por cáncer cérvico-uterino, era tres veces superior en las áreas rurales de México en comparación con las zonas urbanas. ${ }^{12}$ En esta investigación, se intentó no solamente encontrar ese vínculo de "fuerza y dirección" entre las EC y la pobreza o condición marginal en que vive la población enferma, sino además, obtener una unidad de medida para elaborar su ordenamiento y detectar si estaban incluidas en la Lista Oficial, aprobada por el Consejo de Salubridad General en el año 2004.

En México, generalmente, se utilizan dos indicadores para medir las diferencias de desarrollo entre entidades federativas. Un indicador es el Índice de Marginación (IM), construido por el Consejo Nacional de Población (CONAPO) con base en los datos censales $y$, el indicador correspondiente a los conteos de población, elaborados en los años 1995, 2000 y $2005 .{ }^{13}$ El IM, es conceptualmente muy diferente al IDH e incluye cuatro dimensiones estructurales de la marginación, identifica nueve formas de exclusión y mide su intensidad espacial. Aunque se reconoció su probada utilidad, para los propósitos del estudio se utilizó el IDH, pues también es aplicable en la planeación y evaluación de políticas públicas. El IDH difiere conceptualmente del IM, al medir los logros de cualquier sociedad en función de diversos objetivos del desarrollo humano, por ejemplo, la esperanza de vida, la tasa de alfabetización, la tasa bruta de matriculación, el PIB per cápita, entre otros. $^{9}$

En sentido de cálculo, el IDH resalta que sus valores más altos, corresponden a las regiones más desarrolladas, por tanto, se consideró que las EC de mayor prioridad serían las que - dentro del conjunto de las entidades federativas- mostraran una correlación negativa más fuerte, es decir, las que corroboraran la hipótesis de que, a menor desarrollo, corresponde una mayor mortalidad. Hubiera sido útil aplicar la prueba de causalidad o de Granger, ${ }^{8}$ para establecer con mayor precisión si alguna de las variables utilizadas tiene capacidad de predicción sobre la otra y si esta tiene carácter unidireccional o bidireccional, sin embargo, lo impidieron el restringido número de observaciones temporales derivadas de la aplicación del IDH y, los pocos años de implementación, al momento de utilizarse en la investigación. 
No obstante esta limitación, los resultados obtenidos expresaron claro y determinantemente la desigualdad que, en los niveles de desarrollo socioeconómico, existe entre las entidades federativas de México. Los datos disponibles revelan que en 2004, el Distrito Federal (DF) - la entidad más evolucionada- tenía un PIB per cápita de 36 550,30 pesos (a precios de 1993), este monto era 145,24 \% superior al PIB per cápita nacional de 14 903,92 pesos para el mismo año. Por su parte, el estado de Chiapas -el más económicamente rezagado del país- alcanzó un PIB per cápita de 6471,12 pesos, cifra 56,58 \% inferior a dicho índice. ${ }^{14}$

En cuanto a la protección financiera a la salud, como barrera para la desigualdad, la Secretaría de Salud estimó que en 2004 el 45,5 \% de la población del país disponía de algún mecanismo de aseguramiento social, en tanto que en el DF, esta proporción era de $57,4 \%$ y en el estado de Chiapas, de 19,6 \%. Para confirmar estas diferencias entre entidades federativas, también son reveladores, los datos que ofrecen las variables educativas: la tasa de alfabetización alcanza el 97,1 \% para el DF, mientras que en Chiapas la cifra es de 77,1 \%. La tasa de matriculación es de $75,3 \%$ en el caso del DF y de $58,82 \%$ para Guanajuato, que posee la cifra más baja del país.

Ante ese panorama socio-económico, era de esperar una respuesta en la situación epidemiológica de esos territorios y, si se analiza los indicadores más utilizados por su precisión, las diferencias también resultan alarmantes. En ese año 2004, la tasa de mortalidad estandarizada por enfermedades transmisibles, alcanzó el promedio nacional de 71,5 muertes por 100000 habitantes y en el DF, la cifra es de 64,4, o sea, 9,9\%, muy inferior a dicho promedio. ${ }^{15}$ En Chiapas, se notificó una tasa de 112,6 defunciones por 100000 habitantes, 57,5\% superior a la media nacional, certeza del importante rezago de las entidades más empobrecidas, así como del nivel de efectividad de los sistemas de salud y la cobertura de los servicios. Es pertinente enfatizar que, como consecuencia de estas desigualdades regionales, se derivan diferencias significativas en los efectos de las enfermedades no transmisibles (ENT), consideradas catastróficas, como se manifestó en el ejemplo del cáncer cérvico-uterino, lo que contribuye a la prevalencia tanto de estas enfermedades, como de las infecto contagiosas.

Con este trabajo, se aporta elementos válidos para un nuevo enfoque de las desigualdades en salud, en esta ocasión, relacionadas con el carácter catastrófico de las ENT que prevalecen en la compleja realidad nacional y también se estimula a la realización de acciones sanitarias sobre bases sólidas, dirigidas firmemente a los objetivos que hay que compartir.

Resulta inobjetable la vigencia que mantiene el vínculo pobreza-salud, lo que hace impostergable la reorientación social de las políticas y estrategias sanitarias actualmente en ejecución. De otra parte, los procesos de reforma, requieren fundamentarse en sólidas y rigurosas investigaciones que garanticen su estabilidad y con la descripción de esa relación entre el subdesarrollo socio-económico y la presencia de las denominadas enfermedades catastróficas en la República de México, se pretende contribuir a ello. Es loable el reclamo de las autoridades sanitarias mundiales, para recuperar el sentido social de las políticas sanitarias y conseguir los resultados que durante mucho tiempo se ha perseguido y que, hasta ahora, no se alcanza. Es oportuna esa vehemente afirmación de que "estamos, de nueva cuenta, en el punto de partida" y que el desplazamiento rápido y constante de las enfermedades crónicas de las regiones más prósperas, hacia las menos favorecidas, representa un reto de enorme complejidad para los países de ingreso medio y bajo, que se recargan con el mayor peso de estas enfermedades. Enfrentar esa situación, implica riesgos enormes para la tranquilidad financiera de los hogares y, consecuentemente, para la armonía social y la seguridad de las naciones. El 
esfuerzo colectivo, orientado y bien planificado, permitirá aprovechar mejor los recursos - cuantiosos o escasos- de que disponen los países en general y el sector de la salud en particular, para modificar las desiguales condiciones de vida de los ciudadanos y reducir a niveles aceptables, la prevalencia de las enfermedades catastróficas.

\section{REFERENCI AS BI BLI OGRÁFICAS}

1. Tapia Granados J A. Economía y Mortalidad en las Ciencias Sociales: del Renacimiento a las I deas sobre la Transición Demográfia, Rev Salud Colectiva, Buenos Aires. 2005; 1(3): 285-308.

2. OPS/OMS. Los Objetivos de Desarrollo del Milenio en las Américas. Bol Epidemiol. 2004;25(2).

3. Wyszewianski L. Families with catastrophic health care expenditures. Health Service Research. Diciembre 1986 En: Aedo C, editor. Los Problemas de Salud del Adulto Mayor y las Enfermedades Catastróficas. Chile: Estudios Públicos, No. 63; 1996 (Invierno).p. 285-365.

4. Knaul, F. Justicia financiera y gasto catastrófico en salud: impacto del Seguro Popular en México. Rev Salud Pública México. 2005;47(supl 1):57.

5. OPS/OMS. Atención Primaria a la Salud, a 25 años de Alma Ata [serie en Internet]. [citado 1 Sep 2007]. Disponible en:

http://www.paho.org/Spanish/dd/pin/alma-ata_declaracion.htm

6 . Bengoa R. Tendencias recientes y reformas de los sistemas de salud. Anales del Sistema Sanitario de Navarra. 1998; 2(2).

7. Santos Padrón $H$. Relación entre la pobreza, iniquidad y exclusión social con las enfermedades de alto costo en México. Rev Cubana Salud Pública. 2006; 32(2).

8. Gujarati D. Econometría. 4ta ed. México: McGraw Hill; 2005.

9. Cámara de Diputados. Ley General de Salud [serie en Internet]. [citado 1 Sep 2007]. Disponible en: www.diputados.gob. mx/LeyesBiblio/doc/142.doc

10. PNUD. I nforme sobre el desarrollo humano, México 2004.

México: Mundiprensa; 2005.

11. Daniel W. Bioestadística, base para el análisis de las ciencias de la salud. 4ta ed. México: Limusa Wiley; 2002.

12. Palacio-Mejía LS I. Cervical cancer, a disease of poverty: mortality differences between urban and rural areas in Mexico. Salud Publica Mex. 2003; 45 (suppl 3): S315-S325.

13. Consejo Nacional de Población (CONAPO). Índice de Marginación 2005. México: CONAPO; 2006. 
14. Instituto Nacional de Estadística, Geografía e I nformática (I NEGI). Banco de Información Económica [serie en Internet]. [citado 1 Sep 2007]. Disponible en: http://dgcnesyp.inegi.gob. $\mathrm{mx} /$ cgi-win/bdieintsi. exe/Consultar

15. Secretaría de Salud. Sistema Nacional de Información en Salud [serie en Internet]. [citado 1 Sep 2007]. Disponible en:

http:// sinais. salud. gob. $\mathrm{mx} /$ mortalidad/

Recibido: 26 de septiembre de 2007.

Aprobado: 22 de octubre de 2007.

Hilda Santos Padrón. Directora General, Instituto de Seguridad Social de Tabasco (ISSET). México.

\begin{tabular}{|c|c|c|}
\hline orden & $=n f \in r m \in d a d$ & coef, $r$ \\
\hline 1 & Insuficienc a renal crónica & $-0,<76$ \\
\hline$\overline{7}$ & Artritis relumatoide & $-(1, \angle 56$ \\
\hline 3 & Cáncer cérvico uterino & $-0, / 11$ \\
\hline 4 & Insuficierca $a$ reral Ler rmirlal & $-0,260$ \\
\hline 5 & cancer de estomago & $-(1,205$ \\
\hline 6 & Prematurez & $-0,192$ \\
\hline 7 & Ma formaciones conpénita del tubo di jestivn & $-(1,19 ?$ \\
\hline 8 & Infar:o cerebral & $-0,189$ \\
\hline 9 & Cáricer de jróslala & $-0,162$ \\
\hline-0 & Cardıopatias congenitas & $-(1,154$ \\
\hline 1 & Cirrosis hepática & $-0,120$ \\
\hline$?$ & Insa uf. respiratoria del recién nacido & $-(1,115$ \\
\hline .3 & Coma diabétcos & $-0,111$ \\
\hline-4 & LeUCemia y linfomas & $-0,065$ \\
\hline-5 & secuelas de $a \propto c l$ dente vascular & $-(1,(160)$ \\
\hline 6 & Asfixa neonatal & $-0,054$ \\
\hline 7 & Femorragia sıneracnoide.A & $-(1,0,176$ \\
\hline-8 & Femorragia in traencefálica & $-0,018$ \\
\hline .9 & Cáncer del colon y recto & 0,035 \\
\hline 20 & Hngina de secho & $(1,0,5)$ \\
\hline 21 & Cáncer boncogénico y pul nonar & 0.154 \\
\hline 27 & Gepsis neoาetal & $(1,16 \hbar$ \\
\hline 23 & Cáncer de svario & 0,181 \\
\hline 24 & Politraumatizado & 0,186 \\
\hline 25 & Infar:o agldo de migcardio & $(1,198$ \\
\hline 26 & Lupus eritema toso sistémico & 0,205 \\
\hline 27 & otros rrob emas respiratorics del rerien nadido & $(1,218$ \\
\hline 28 & Déectos diafragmáticcs & 0,212 \\
\hline 29 & $\mathrm{VIH} / \mathrm{SIDA}$ & 0,315 \\
\hline 30 & cancer de nama & 0,327 \\
\hline
\end{tabular}


IDH Estatal

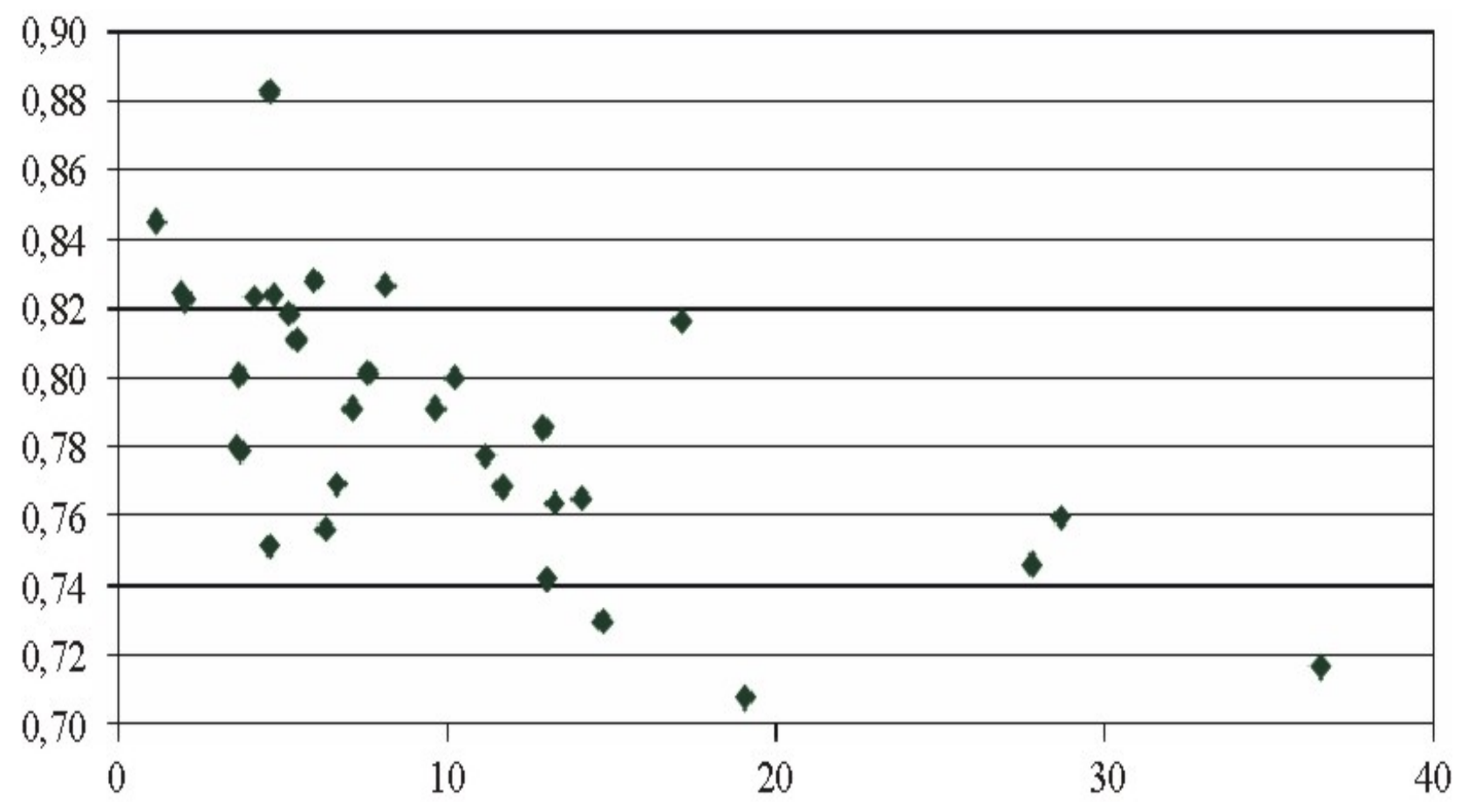

Tasa de mortalidad estatal (x 1,000,000 hab)

Fuentes: SEED, Secretaría de Salud/IDH, CONAPO. Fig. Gráfico de dispersión entre Índice de Desarrollo Humano (IDH) y tasas de mortalidad por cáncer cérvico-uterino de las entidades federativas (2004). 